\title{
Preface
}

\section{The Facial Skin Defect: A Reconstructive Opportunity}

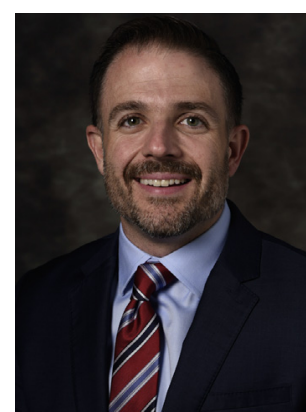

Anthony M. Bunnell, MD, DMD, FACS Editor
I am honored and grateful for the opportunity to introduce this issue of the Atlas of the Oral and Maxillofacial Surgery Clinics of North America devoted to reconstruction of facial skin defects. Each day, the maxillofacial surgeon is faced with many challenges and options in reconstructing facial skin defects. The key is to select the most appropriate reconstructive method and setting for this to take place. This may be a daunting task, but when equipped with the proper tools, a reconstructive challenge can turn into an opportunity to produce an aesthetically pleasing reconstructive outcome. The goal of this issue of the Atlas of the Oral and Maxillofacial Surgery Clinics of North America is to provide the tools necessary for the reader to develop a surgical armamentarium of the most commonly used local flaps in facial reconstruction.

The reader will find that each article within this Atlas of the Oral and Maxillofacial Surgery Clinics of North America is dedicated to a single flap for an in-depth review of flap design and execution. Pertinent surgical anatomy as well as a "how-to" explanation of flap elevation is incorporated into each article to provide the reader a comprehensive understanding on each flap described. The authors have also offered their expertise by sharing clinical applications through case examples as well as surgical pearls and pitfalls one may encounter during the reconstructive process.

I would be remiss if I did not acknowledge the hard work and dedication of the authors in order to produce this Atlas of the Oral and Maxillofacial Surgery Clinics of North America. It has been a great privilege to work with these authors, and I express my gratitude for their time and contributions in producing these informative articles. Thank you to Dr. Rui Fernandes, the Consulting Editor, for his guidance and support of this project. I also want to thank my mentors, colleagues, fellows, and residents at the University of Florida, who continue to inspire me to be a better surgeon and teacher each day. Lastly, I want to acknowledge the endless support from my family in allowing me to pursue this endeavor.

It is my hope that the readers will find this Atlas of the Oral and Maxillofacial Surgery Clinics of North America a useful tool in facial reconstruction and will provide their patients the best reconstructive outcome.

Anthony M. Bunnell, MD, DMD, FACS Division of Head and Neck Surgery Department of Oral and Maxillofacial Surgery University of Florida College of Medicine-Jacksonville 653-1 West 8th Street Jacksonville, FL 32209, USA

E-mail address: anthony.bunnell@jax.ufl.edu 